\title{
The Classification of T Dwarfs
}

\author{
Adam J. Burgasser ${ }^{1}$, J. Davy Kirkpatrick ${ }^{2}$, and Michael E. Brown ${ }^{1}$ \\ ${ }^{1}$ California Institute of Technology, MSC 103-33, Pasadena CA 91125, USA \\ 2 Infrared Processing and Analysis Center, MSC 100-22, Pasadena CA 91125, USA \\ "...one is forced to wonder where it will lead to, if everyone who works on stellar \\ spectra also introduces a new classification..." \\ - Nils Duner (1899)
}

\begin{abstract}
We discuss methods for classifying T dwarfs based on spectral morphological features and indices. $\mathrm{T}$ dwarfs are brown dwarfs which exhibit methane absorption bands at 1.6 and $2.2 \mu \mathrm{m}$. Spectra at red optical $(6300-10100 \AA)$ and near-infrared $(1-2.5 \mu \mathrm{m})$ wavelengths are presented, and differences between objects are noted and discussed. Spectral indices useful for classification schemes are presented. We conclude that near-infrared spectral classification is generally preferable for these cool objects, with data sufficient to resolve the 1.17 and $1.25 \mu \mathrm{m} \mathrm{K}$ I doublets lines being most valuable. Spectral features sensitive to gravity are discussed, with the strength of the Kband peak used as an example. Such features may be used to derive a two-dimensional scheme based on temperature and mass, in analogy to the MK temperature and luminosity classes.
\end{abstract}

\section{Introduction}

In the last decade, we have witnessed a flood of low-mass star and brown dwarf detections, due to improvements in infrared array technologies and the advent of large-scale, near-infrared sky surveys using these detectors. In 1995, the first brown dwarfs were discovered after decades of failed searches - the companion object Gl 229B [31] and the Pleiades brown dwarf Teide 1 [36]. Since then, nearly one hundred brown dwarfs have been detected as companions to nearby stars and in the field, most made by new sky surveys such as the Two Micron All Sky Survey (2MASS [40]), the Sloan Digital Sky Survey (SDSS 433), and the Deep Near-Infrared Survey (DENIS [12]). Others have been identified photometrically in various cluster surveys (see contribution by R. Rebolo, these proceedings). These surveys have finally brought brown dwarfs out of the realm of the theoretical and into that of the observational.

Two new spectral classes have also been defined as a result of these low-mass discoveries. The $L$ spectral class, comprised of objects cooler than $\mathrm{M} 9.5 \mathrm{~V}$, is defined by [19] and is discussed in the contribution by J.D. Kirkpatrick in these proceedings. The $T$ spectral class, defined by the presence of $\mathrm{CH}_{4}$ absorption at $2.2 \mu \mathrm{m}$ 19], is discussed here. The prototype for this class, Gl 229B, shows $\mathrm{CH}_{4}$ and $\mathrm{H}_{2} \mathrm{O}$ absorption bands throughout the near-infrared [34,35], exhibiting a spectral morphology similar to those of the giant planets [14]. Gl 229B remained 
Adam J. Burgasser, et al.

the solitary T dwarf until mid-1999, when 2MASS, SDSS, and the New Technology Telescope Deep Field uncovered field analogs. To date, at least $23 \mathrm{~T}$ dwarfs have been identified [3, $1,6,6,1,42,24$, a number sufficient to begin consideration of spectral classification.

\subsection{Spectral Classification of Cool Dwarfs}

The classification of late-type dwarfs (which we define here as objects later than spectral type $\mathrm{K} 7 \mathrm{~V}$ ) has been done for over one hundred years using a variety of features in photographic, red optical, and near-infrared spectra. Figure 1 diagrams the wavelength and spectral type ranges of a few major classification schemes related to the widely accepted MK process. The classification of M dwarfs began with the conversion of Secchi's type III stars 39] to class M by W. Fleming in the Henry Draper catalog [32]. This class was further divided by A.J. Cannon into subtypes $\mathrm{Ma}, \mathrm{Mb}, \mathrm{Mc}$, and $\mathrm{Md}$, based on their spectral morphologies in the photographic regime 10. Work by several authors using different instrumental configurations extended $\mathrm{M}$ dwarf classification to decimal systems up to type M6 16, 22, 23,30, 17, based primarily on the strength of $\mathrm{TiO}$ bands as temperature discriminants. Classification up to type M9V was done by P.C. Boeshaar in the photographic [1,2] and J.D. Kirkpatrick in the red optical [21,20], based on the appearance of $\mathrm{TiO}, \mathrm{VO}$, and $\mathrm{CaOH}$ bands. The latter scheme also makes use of spectral slope from $6300-9000 \AA$ as an additional temperature discriminant. Following the discovery of numerous objects later than type M9.5V, J.D. Kirkpatrick defined and classified the L spectral class in the red optical based on the appearance of weakening oxides and strengthening metal hydride bands and alkali lines [19]. Classification of the L class in the near-infrared is currently under investigation by various authors. The MK(K) system 30,17] makes use of additional spectral features (e.g., $\mathrm{CaOH}, \mathrm{Na} \mathrm{D}$ lines) as luminosity discriminants in order to create a two-dimensional scheme. Luminosity class distinctions have not been made for $\mathrm{L}$ dwarfs, as the coolest giant stars retain strong $\mathrm{TiO}$ and $\mathrm{VO}$ bands [19].

The schemes for $\mathrm{T}$ dwarf classification shown as dashed lines in Fig. 1 are the focus of this article, and are discussed more fully in [7]. In $\S 2$ we address red optical classification, based on a spectral sample of bright $\mathrm{T}$ dwarfs identified by 2MASS and SDSS. In $\S 3$ we discuss near-infrared classification of a larger sample, and discuss spectral features important for classification. In $\S 4$ we describe spectral features in the near-infrared which may depend on specific gravity, and could therefore append a second dimension to a temperature scheme, in analogy to the MK luminosity classes. We summarize our discussion in $\S 5$.

\section{Red Optical Classification}

L dwarfs are classified by 19 using 6300-10100 $\AA$ red optical spectra at $9 \AA$ resolution, obtained with the Low Resolution Imaging Spectrograph (LRIS [33]) mounted on the Keck 10m Telescope. As shown in Fig. 6 of [19], this spectral 
range samples most of the prominent molecular and atomic features in late-M and L dwarf spectra, and a "recipe" for spectral classification from M7V to L8V is presented based on measurements of spectral indices on selected standards. In order to make a direct comparison with this L dwarf scheme, we have obtained optical spectra for nine $\mathrm{T}$ dwarfs utilizing the same instrumental setup as [19]. Data acquisition and reduction are described in [5.,7]. Reduced spectra are shown in Fig. 2 for 2MASS 0559-14 [4 and Gl 570D [4, along with data for Gl 229B [35 and L dwarfs 2MASS 1507-16 (L5V), 2MASS 0920+35 (L6.5V), and 2MASS $1632+19(\mathrm{~L} 8 \mathrm{~V})$ 19. 18]. Spectra are displayed on a log scale and offset in order to highlight features.

A few general trends are readily apparent. Most obvious is the increased spectral slope from $8000-10100 \AA$ in the $\mathrm{T}$ dwarfs, a feature which has been attributed to pressure broadened K I at 7665 and $7699 \AA$ [9,25]. A drop in flux shortward of $6600 \AA$ is due to the similarly broadened Na I D lines at 5890 and $5895 \AA$ [37. Increased $\mathrm{H}_{2} \mathrm{O}$ absorption at $9250 \AA$ is seen in the $\mathrm{T}$ dwarfs, strongest in Gl 229B and Gl 570D. CrH (8611 $\AA), \mathrm{FeH}(8692 \AA)$, and Na I (8183, $8195 \AA$ ) features weaken in the late L dwarfs, and are completely absent in the T dwarfs. The behavior of the Cs I $(8543,8943 \AA)$ lines is less straightforward, appearing to strengthen slightly from L5V to 2MASS 0559-14, but weakening in Gl 229B and Gl 570D, with the $8943 \AA$ line becoming blended with $\mathrm{CH}_{4}$ at $8900 \AA$. Rb I (7800, $7948 \AA$ ) lines may be strengthening from mid-L to 2MASS 0559-14, but lack of signal prevents their detection in the other $\mathrm{T}$ dwarfs (only five objects in our sample exhibit flux shortward of $8000 \AA)$. FeH $(9896 \AA)$ is known to weaken from mid- to late-L [19], and is barely detected in 2MASS 1632+19; however, 2MASS 0559-14 shows a clear band, while Gl 229B and Gl $570 \mathrm{D}$ do not. $\mathrm{CaH}(6750 \AA)$ may be present in all of the spectra shown here, but is exceedingly weak in the latest L dwarfs and 2MASS 0559-14.

It is clear, then, that there are qualitative differences in the optical spectra of $\mathrm{L}$ and $\mathrm{T}$ dwarfs. The $\mathrm{T}$ dwarfs themselves appear to be distinguishable, based on their spectral slope, the presence of $9896 \AA \mathrm{FeH}$, and the $9250 \AA \mathrm{H}_{2} \mathrm{O}$ band strength. To explore these differences quantitatively, we have examined a set of spectral indices, as described in Table 1. Index values are plotted in Fig. 3 for the five brightest $\mathrm{T}$ dwarfs in our sample and Gl 229B (open circles), as well as late-M and L dwarf standards from 19] (solid circles). T dwarfs are ordered by their $\mathrm{H}_{2} \mathrm{O}$ index. The deepening of the $9250 \AA \mathrm{H}_{2} \mathrm{O}$ band in the $\mathrm{T}$ dwarfs is readily apparent, as is the increased spectral slope measured by the Color- $\mathrm{d}$ index. Weakening of the $8611 \AA \mathrm{CrH}$ and $9896 \AA \mathrm{FeH}$ bands from types L to $\mathrm{T}$ is similarly seen in their respective indices. The KI-a index, which measures the relative depth of the broadened $\mathrm{K}$ I doublet (or alternately its breadth), peaks at around $\mathrm{L} 8 \mathrm{~V}$, then decreases through the $\mathrm{T}$ dwarfs; this is due either to lower signal-to-noise in the latter objects or the formation of $\mathrm{KCl}$ at $\mathrm{T}_{\text {eff }} \sim 950 \mathrm{~K}$ [26. Cs I ( $8543 \AA)$ shows a general strengthening but with significant scatter, reflecting its observed ambiguous behavior.

Based on these spectral indices and the quality of the observed spectra, we make three conclusions regarding $\mathrm{T}$ dwarf classification in the optical: 
Table 1. T Dwarf Red Optical Spectral Indices

\begin{tabular}{clll}
\hline Index & Numerator $(\AA)$ & Denominator $(\AA)$ & Feature Measured \\
\hline KI-a & $7100.0-7300.0$ & $7600.0-7800.0$ & $7665,7679 \AA$ K I doublet \\
Cs-a & Ave. 8496.1-8506.1 and 8536.1-8546.1 & $8516.1-8526.1$ & $8543 \AA$ Cs I line \\
$\mathrm{CrH}-\mathrm{a}$ & $8580.0-8600.0$ & $8621.0-8641.0$ & $8611 \AA \mathrm{CrH}$ band $^{a}$ \\
$\mathrm{H}_{2} \mathrm{O}$ & $9225.0-9250.0$ & $9275.0-9300.0$ & $9250 \AA \mathrm{H}_{2} \mathrm{O}$ band $^{a}$ \\
FeH-b & $9863.0-9883.0$ & $9908.0-9928.0$ & $9896 \AA \mathrm{FeH}$ band $^{a}$ \\
Color-d & $9675.0-9875.0$ & $7350.0-7550.0$ & spectral slope $^{a}$ \\
\hline
\end{tabular}

${ }^{a}$ Also used as indices for L dwarfs in [19].

- L and T dwarfs (later than 2MASS 0559-14) appear to be distinguishable in the red optical, due to the absence of $\mathrm{CrH}(8611 \AA)$ and $\mathrm{FeH}(8692 \AA)$ bands, increased $9250 \AA \mathrm{H}_{2} \mathrm{O}$ absorption, and increased spectral slope in the T dwarfs.

- T dwarfs can be separated in the red optical based on the presence or absence of the $9896 \AA \mathrm{FeH}$ band, $9250 \AA \mathrm{H}_{2} \mathrm{O}$ band strength, and spectral slope. The observed scatter, however, restricts us to only distinguishing "early" $\mathrm{T}$ dwarfs (e.g., 2MASS 0559-14) from "late" ones (e.g., Gl 570D).

- Despite these successes, the faintness of these objects in the optical $\left(\mathrm{M}_{R} \sim\right.$ 24.6 for Gl 229B [15]) makes it necessary to consider classification in the near-infrared.

\section{Near-Infrared Classification}

The spectral energy distribution of $\mathrm{T}$ dwarfs peaks at around $1.27 \mu \mathrm{m}$, the center of the J-band window, due to the combined effects of temperature and $\mathrm{H}_{2} \mathrm{O}$, $\mathrm{CH}_{4}$, and collision-induced (CIA) $\mathrm{H}_{2}$ absorption features. The 1-2.5 $\mu \mathrm{m}$ region also encompasses the defining $2.2 \mu \mathrm{m} \mathrm{CH}_{4}$ band. In our program to identify these cool brown dwarfs, we have acquired 1-2.5 $\mu \mathrm{m}$ spectra of $14 \mathrm{~T}$ dwarfs at a resolution of $\mathrm{R} \sim 100(100 \AA$ at $1 \mu \mathrm{m})$ using the Near-Infrared Camera (NIRC [28]) mounted on the Keck 10m Telescope. Instrumental setup and data reduction are described in [7]. Figure 4 displays these spectra (thick lines), along with NIRC data for Gl 229B [35], and SDSS T dwarf data from the literature 4124] (higher resolution thin lines). A NIRC spectrum of 2MASS 0920+35 $(\mathrm{L} 6.5 \mathrm{~V})$ is shown for comparison. All spectra are normalized at their J-band peaks.

The spectra in Fig. 4 are ordered by visual inspection, based on the increasing strengths of the 1.15 and $1.6 \mu \mathrm{m}$ absorption bands. The $1.15 \mu \mathrm{m}$ feature begins in the late $\mathrm{L}$ dwarfs as a blend of $\mathrm{H}_{2} \mathrm{O}$, two $\mathrm{K}$ I doublets $(1.169$ \& 1.177, 1.243 
\& $1.252 \mu \mathrm{m}), \mathrm{Na} \mathrm{I}(1.138,1.141 \mu \mathrm{m})$, and $\mathrm{FeH}(1.19,1.21,1.24 \mu \mathrm{m})$ 29], but is later influenced by $\mathrm{CH}_{4}$ absorption at $1.1 \mu \mathrm{m}$ in the $\mathrm{T}$ dwarfs. The deepening and broadening of this feature and the adjacent $\mathrm{H}_{2} \mathrm{O} / \mathrm{CH}_{4}$ absorption at $1.4 \mu \mathrm{m}$ causes the J-band flux to become more peaked and narrow toward the latest objects shown. The $1.15 \mu \mathrm{m}$ feature also depresses the $1.243 \& 1.252 \mu \mathrm{m} \mathrm{K}$ I doublet, unresolved in the NIRC data. There is significant suppression of the H- and K-band peaks between SDSS 1021-03 and 2MASS 2254+31, coinciding with a large near-infrared color difference between these objects $(\mathrm{J}-\mathrm{H} \sim 0.5 \mathrm{mag}$ redder in SDSS 1021-03). This occurs after CO at $2.3 \mu \mathrm{m}$, a prominent feature in late-M and $\mathrm{L}$ dwarfs, disappears. Toward later types, the K-band peak shape evolves from two bandheads at $2.2\left(\mathrm{CH}_{4}\right)$ and $2.3 \mu \mathrm{m}(\mathrm{CO})$, to a rounded peak with a kink at $2.17 \mu m$, to a symmetric peak at 2.07 $\mu \mathrm{m}$ in 2MASS 0415-09.

In order to quantitatively explore these observed spectral variations, we have defined near-infrared spectral indices in analogy to those derived for the red optical spectra. These indices are described in Table 3 and plotted in Fig. 5 for the NIRC data (circles), along with values computed from L and T dwarf spectral data from the literature 14,41,13,24 (triangles). Filled and open symbols denote $\mathrm{L}$ and $\mathrm{T}$ dwarfs, respectively. $\mathrm{T}$ dwarfs are ordered as in Fig. 4, while $\mathrm{L}$ dwarfs are ordered by their spectral type from [19]. Aligned symbols represent multiple spectra for the same object, allowing us to estimate the approximate noise in these values, as well as compare spectra of different resolutions. There is an obvious correlation between the depth of the $1.15 \mu m \mathrm{H}_{2} \mathrm{O} / \mathrm{CH}_{4}$ band, the $1.3 \mu \mathrm{m} \mathrm{CH}_{4}$ band wing, and the $1.6 \mu \mathrm{m} \mathrm{CH}_{4}$ band, all of which steadily increase from the late-L dwarfs through 2MASS 0415-09. The K-shape index (measuring the change of the $2.17 \mu \mathrm{m}$ kink) also increases toward unity (no kink) over this range, although with more scatter. Similarly, the J/K peak ratio appears to generally increase, although there is less agreement between the NIRC data and data from the literature. One object, 2MASS 0937+29 (see below) is particularly discrepant. Finally, the $\mathrm{J} / \mathrm{z}$ peak ratio shows the least correlation with morphological order, with variations no more than $40 \%$ of the mean, suggesting noise as a possible contributor.

Overall, their appears to be a good correlation in the strengths of the $\mathrm{CH}_{4}$ and $\mathrm{H}_{2} \mathrm{O}$ absorption band strengths, and these are likely to be crucial features for spectral typing in the near-infrared. K I lines, unresolved in this data, are also important indicators of temperature (being higher energy lines than the broadened $7665 \& 7699 \AA \mathrm{K}$ I doublet in the red optical), so that classification spectra in this wavelength regime should be of sufficient resolution to permit measurement of these lines. These data can be easily obtained using existing instrumentation.

\section{Additional Classification Parameters in the Near-Infrared}

The discrepancy seen in the $\mathrm{J} / \mathrm{K}$ peak ratio for 2 MASS $0937+29$ is fairly obvious in Fig. 5, and can be seen in Fig. 6a, which shows the 1.5-2.5 $\mu \mathrm{m}$ spectra of 
Table 2. T Dwarf Near-Infrared Spectral Indices

\begin{tabular}{llll}
\hline Index & Numerator $(\mu m)$ & Denominator $(\mu m)$ & Feature Measured \\
\hline $\mathrm{zJ}-\mathrm{H}_{2} \mathrm{O}$ & $1.25-1.28$ & $1.13-1.16$ & $1.15 \mu m \mathrm{H}_{2} \mathrm{O} / \mathrm{CH}_{4}$ band \\
$\mathrm{J} / \mathrm{z}$ & $1.26-1.28$ & $1.06-1.08$ & J-band $/ \mathrm{z}$-band peak ratio \\
$\mathrm{J}-\mathrm{CH}_{4}$ & $1.26-1.275$ & $1.295-1.31$ & $1.3 \mu m \mathrm{CH}_{4}$ absorption wing \\
$\mathrm{H}_{-} \mathrm{CH}_{4}$ & $1.57-1.59$ & $1.63-1.65$ & $1.6 \mu m \mathrm{CH}_{4}$ band \\
$\mathrm{J} / \mathrm{K}$ & $1.26-1.28$ & $2.06-2.09$ & $\mathrm{~J}$-band $/ \mathrm{K}$-band peak ratio \\
$\mathrm{K}-$ shape & $2.10-2.11$ minus $2.16-2.17$ & $2.17-2.18$ minus $2.19-2.20$ & K-band shape $(2.17 \mu m$ kink $)$ \\
\hline
\end{tabular}

2MASS $0937+29$ and 2MASS $1047+21$, both normalized at their J-band peaks. The similarity of these objects at H-band is in striking contrast to the notable suppression of the K-band peak of 2MASS $0937+29$. This object is the bluest T dwarf so far identified, with $\mathrm{J}-\mathrm{K}_{s}=-0.89 \pm 0.24$, as compared to the mean $\mathrm{J}-\mathrm{K}_{s}$ $\sim 0$ for all other 2MASS T dwarfs. The K-band peak is shaped by the combined effects of $\mathrm{H}_{2} \mathrm{O}(1.8-2.1 \mu \mathrm{m}), \mathrm{CH}_{4}(2.2-2.6 \mu \mathrm{m})$, and CIA $\mathrm{H}_{2}$ (centered around 2.5 $\mu \mathrm{m}$ ). The latter feature is strongest at K-band, and, being a collisional process, is highly sensitive to the total photospheric pressure. Zero-metallicity models show that increased gravity (and therefore photospheric temperature) results in bluer $\mathrm{H}-\mathrm{K}$ colors due to increased $\mathrm{H}_{2}$ absorption, an effect which becomes more pronounced toward cooler $\mathrm{T}_{\text {eff }}[38$.

To examine this effect, we have computed spectral indices for models provided by D. Saumon (priv. comm.). Figure $6 \mathrm{~b}$ shows the behavior of the $\mathrm{J} / \mathrm{K}$ peak ratio as compared to $1.6 \mu \mathrm{m} \mathrm{CH}_{4}$ band strength for gravities of 300,1000 , and $3000 \mathrm{~m} \mathrm{~s}^{-2}$. Overplotted are index values measured for the 2MASS T dwarfs (open circles). Gl 570D is separately indicated (solid triangle); this companion object has an estimated $\mathrm{T}_{\text {eff }}=750 \pm 50 \mathrm{~K}$ and $\mathrm{g}=800-2000 \mathrm{~m} \mathrm{~s}^{-2}$ 朋, in consensus with its location in Fig. $6 \mathrm{~b}$ relative to the models. The large spread in $\mathrm{J} / \mathrm{K}$ index values versus gravity for the models is obvious, and is greater at lower temperatures. We see that 2MASS 0937+29 and 2MASS 1047+21 (solid circles) lie on opposite sides of the model $\mathrm{g}=3000 \mathrm{~m} \mathrm{~s}^{-2}$ line, consistent with the former having a higher surface gravity. This example suggests that comparison of the K-band peaks for a sample of classified $\mathrm{T}$ dwarfs may allow segregation by gravity (and therefore mass), in analogy to the dwarf/giant segregation made in the MK luminosity classes. Variations in metallicity may also play an important role in $\mathrm{H}_{2}$ strength, and the effects of CIA $\mathrm{H}_{2}$ absorption at other wavelengths (i.e., near peaks at 0.83 and $1.25 \mu \mathrm{m}$ ) needs to be similarly investigated. Alkali lines, particularly the 1.17 and $1.25 \mu \mathrm{m} \mathrm{K}$ I doublets, may also elucidate gravity and/or metallicity effects, and provide a further discriminant for a twodimensional classification scheme. 


\section{Summary}

We have discussed the prospects for spectral typing $\mathrm{T}$ dwarfs in the red optical and near-infrared, based on analysis of spectral data obtained by the authors and from the literature. Basic morphological differences are seen in both regimes, supporting the viability of considering classification schemes for these cool brown dwarfs. Several conclusions may be drawn from the preceding discussion:

- The red optical spectra of T dwarfs is different than that of the $\mathrm{L}$ dwarfs, due to differences in spectral slope, $9250 \AA \mathrm{H}_{2} \mathrm{O}$ band strength, and the presence or absence of various hydride bands and alkali lines. The faintness of the $\mathrm{T}$ dwarfs at these wavelengths, however, favors classification schemes at longer wavelengths.

- A continuous sequence of $\mathrm{T}$ dwarfs can be seen in near-infrared spectral data, both at low $(\mathrm{R} \sim 100)$ and moderate $(\mathrm{R} \sim 600)$ resolutions. This sequence is dominated by the bands of $\mathrm{CO}$ (in the earliest T dwarfs), $\mathrm{H}_{2} \mathrm{O}, \mathrm{CH}_{4}$, and CIA $\mathrm{H}_{2}$, while the $1.25 \mu \mathrm{m} \mathrm{K}$ I doublet, unresolved in the NIRC data, may show a corresponding progression. The clear variation in features and greater facility of obtaining reasonable signal-to-noise spectra favors this wavelength regime for classification.

- Spectral features that are sensitive to specific gravity may allow development of a two-dimensional scheme analogous to the MK luminosity class, but instead based on object mass. The strength of the K-band peak appears to be such a feature, due to pressure-sensitive CIA $\mathrm{H}_{2}$ absorption. Calibration of this effect and the influence of metallicity remains to be explored.

A.J. Burgasser acknowledges S.K. Leggett, B.R. Oppenheimer, and M.A. Strauss for providing useful spectral data for comparison, and D. Saumon for use of unpublished model spectra. Enlightening discussions over the role of $\mathrm{H}_{2}$ were made with A. Burrows, M. Marley, and D. Saumon. AJB also acknowledges the contributions of the other members of the 2MASS Rare Objects Team: R. Cutri, C. Dahn, J. Gizis, J. Liebert, B. Nelson, and I.N. Reid. Data presented herein were obtained at the W. M. Keck Observatory which is operated as a scientific partnership among the California Institute of Technology, the University of California, and the National Aeronautics and Space Administration. The Observatory was made possible by generous financial support of the W. M. Keck Foundation. This contribution makes use of data from the Two Micron All Sky Survey, which is a joint project of the University of Massachusetts and the Infrared Processing and Analysis Center, funded by the National Aeronautics and Space Administration and the National Science Foundation.

\section{References}

1. P.C. Boeshaar: The spectral classification of M-dwarf stars. PhD Thesis, Ohio State University, Columbus (1976)

2. P.C. Boeshaar, \& J.A. Tyson: AJ 90, 817 (1985) 
Adam J. Burgasser, et al.

3. A.J. Burgasser, et al.: ApJ 522, L65 (1999)

4. A.J. Burgasser, et al.: ApJ 531, L57 (2000a)

5. A.J. Burgasser, J.D. Kirkpatrick, I.N. Reid, J. Liebert, J.E. Gizis, \& M. Brown: AJ 120, 473 (2000b)

6. A.J. Burgasser, et al.: ApJ 120, 1100 (2000c)

7. A.J. Burgasser, et al.: ApJ (2001) in prep.

8. A. Burrows, et al.: ApJ 491, 856 (1997)

9. A. Burrows, M.S. Marley, C.M. Sharp: ApJ 531, 438 (2000)

10. A.J. Cannon, \& E.C. Pickering: Ann. Astron. Obs. Harvard Coll. 28(II), 131 (1901)

11. J.G. Cuby, P. Saracco, A.F.M Moorwood, S. D’Odorico, C. Lidman, F. Comerón, \& J. Spyromilio: A\&A 349, L41 (1999)

12. N. Epchtein, et al.: In Science with Astronomical Near-Infrared Sky Surveys. ed. by N. Epchtein, A. Omont, B. Burton B., \& P. Persi (Kluwer, Dordrecht 1994) p. 3

13. X. Fan, et al.: AJ 119, 928 (2000)

14. T.R. Geballe, S.R. Kulkarni, C.E. Woodward, \& G.C. Sloan: ApJ 467, L101 (1996)

15. D.A. Golimowski, C.J. Burrows, S.R. Kulkarni, B.R. Oppenheimer, \& R.A. Brukardt: AJ 115, 2579 (1998)

16. D. Hoffleit: Ann. Astron. Obs. Harvard Coll. 105, 45 (1937)

17. H.L. Johnson, \& W.W. Morgan: ApJ 117, 313 (1953)

18. J.D. Kirkpatrick, I.N. Reid, J. Liebert, J.E. Gizis, A.J. Burgasser, D.G. Monet, C.C. Dahn, B. Nelson, \& R.J. Williams: AJ 120, 447 (2000)

19. J.D. Kirkpatrick, et al.: ApJ 519, 802 (1999)

20. J.D. Kirkpatrick, T.J. Henry, \& M.J. Irwin: AJ 113, 1421 (1997)

21. J.D. Kirkpatrick, T.J. Henry, \& D.W. McCarthy: ApJ Supp. 77, 417 (1991)

22. G.P. Kuiper: ApJ 88, 429 (1938)

23. G.P. Kuiper: ApJ 95, 201 (1942)

24. S.K. Leggett, et al.: ApJ 536, L35 (2000)

25. J. Liebert, I.N. Reid, A. Burrows, A.J. Burgasser, J.D. Kirkpatrick, \& J.E. Gizis: ApJ 533, L155 (2000)

26. K. Lodders: ApJ 519, 793 (1999)

27. E.L. Martín, X. Delfosse, G. Basri, B. Goldman, T. Forveille, \& M.R. ZapateroOsorio: AJ, 118, 2466 (1999)

28. K. Matthews, \& B.T. Soifer: In Infrared Astronomy with Arrays: The Next Generation. ed. by I. McLean (Dordrecht, Kluwer 1994) p. 239

29. I.S. McLean, et al.: ApJ, 533, L45 (2000)

30. W.W. Morgan, P.C. Keenan, \& E. Kellman: An Atlas of Stellar Spectra, with an Outline of Spectral Classification. (Chicago, Univ. Chicago Press 1943)

31. T. Nakajima, B.R. Oppenheimer, S.R. Kulkarni, D.A. Golimowski, K. Matthews, \& S.T. Durrance: Nature 378, 463 (1995)

32. E.C. Pickering: Ann. Astron. Obs. Harvard Coll. 27, 1 (1890)

33. J.B. Oke, et al.: PASP 107, 375 (1995)

34. B.R. Oppenheimer, S.R. Kulkarni, K. Matthews, \& T. Nakajima; Science 270, 1478 (1995)

35. B.R. Oppenheimer, S.R. Kulkarni, K. Matthews, \& M.H. van Kerkwijk: ApJ 502, 93 (1998)

36. R. Rebolo, M.R. Zapatero-Osorio, \& E.L. Martín: Nature 377, 129 (1995)

37. I.N. Reid, et al.: ApJ 521, 631 (1999)

38. D. Saumon, P. Bergeron, J.I. Lunine, W.B. Hubbard, \& A. Burrows: ApJ 424, 333 (1994) 
39. A. Secchi: CR Acad. Sci. Paris 63, 621 (1866)

40. M.F. Skrutskie, et al.: In The Impact of Large-Scale Near-IR Sky Surveys. ed. by F. Garzon (Dordrecht, Kluwer 1997) p. 25

41. M.A. Strauss, et al: ApJ 522, L61 (1999)

42. Z.I. Tsvetanov, et al: ApJ 531, L61 (2000)

43. D.G. York, et al: AJ 120, 1579 (2000) 


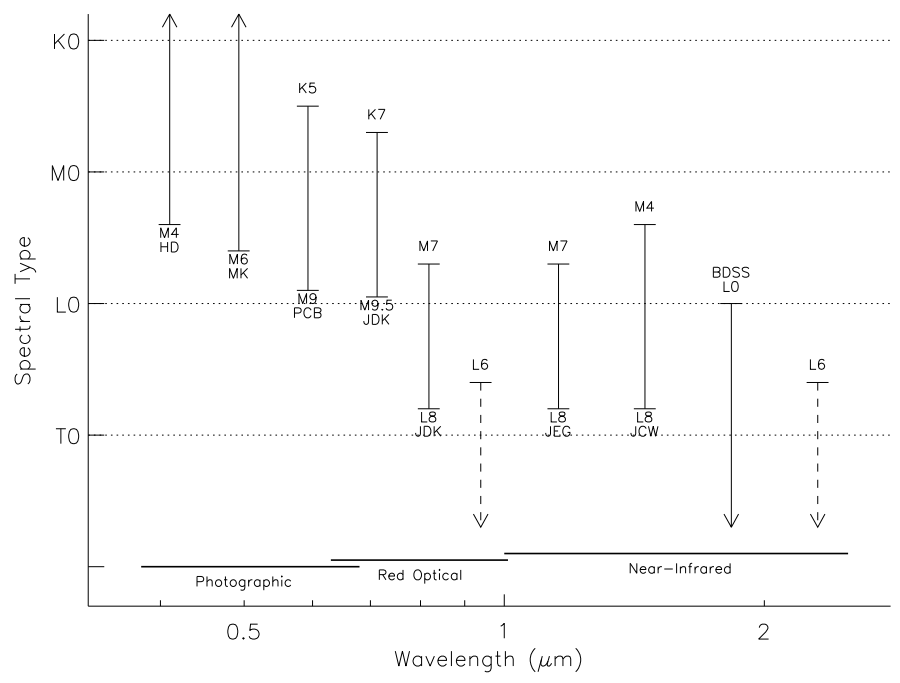

Fig. 1. Spectral typing schemes for M, L and T dwarfs over the past century. The HD scheme is that of the Henry Draper catalog [32,10,16]; the MK (Yerkes) scheme is described in 22,23,30,17; $\mathrm{PCB}$ are the P.C. Boeshaar schemes of 1, 2]; JDK are the $\mathrm{M}$ and L dwarf schemes of J.D. Kirkpatrick 221,20,19]; near-infrared schemes for L dwarfs are for J.E. Gizis (priv. comm.), J.C. Wilson (priv. comm.), and the NIRSPEC Brown Dwarf Spectroscopic Survey (I. McLean; priv. comm.); the dashed lines represent spectral schemes discussed in the text, also described in [7]. 


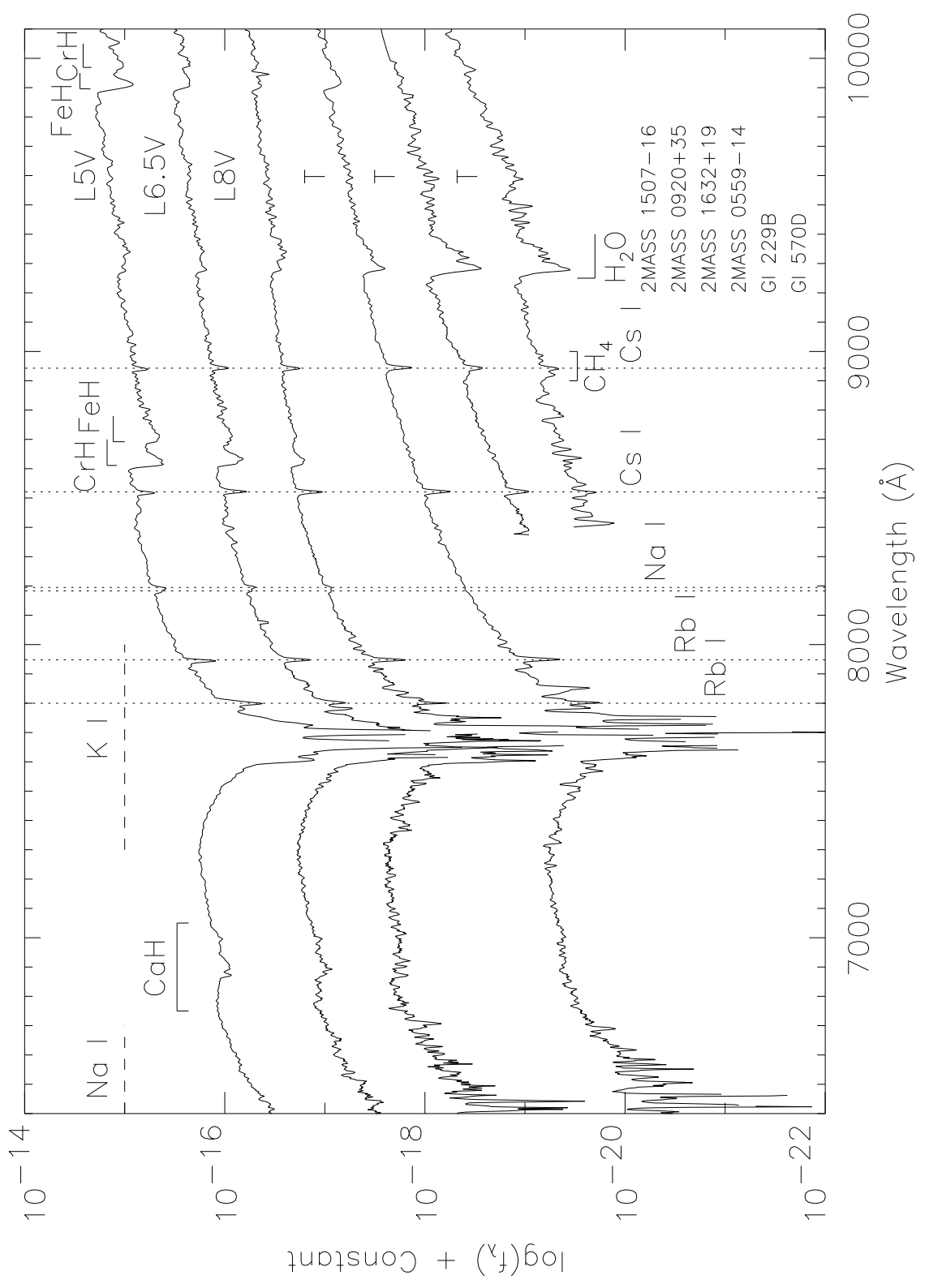

Fig. 2. Red optical spectra of $\mathrm{L}$ and $\mathrm{T}$ dwarfs. Spectra are displayed on a log scale and offset by a constant factor. Major molecular and atomic features are noted. L dwarf spectra are from [19,18], Gl 229B data from 35]. 

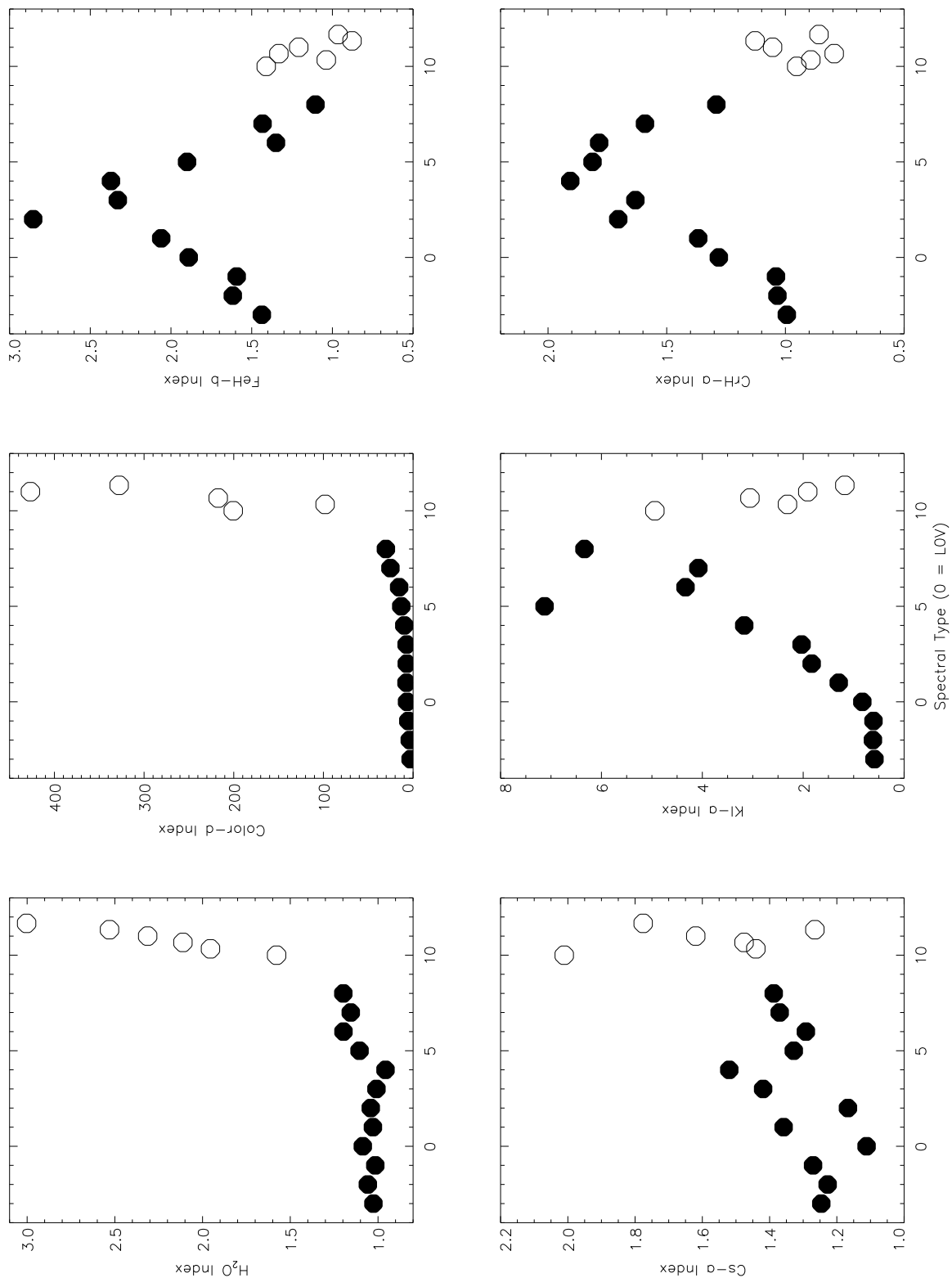

Fig. 3. Red optical spectral indices for late-M, L, and T dwarfs. M and L dwarf values (filled circles) are calculated using data from [19.18. T dwarfs (open circles) are ordered by their $\mathrm{H}_{2} \mathrm{O}$ index. Indices are described in Table 1. 

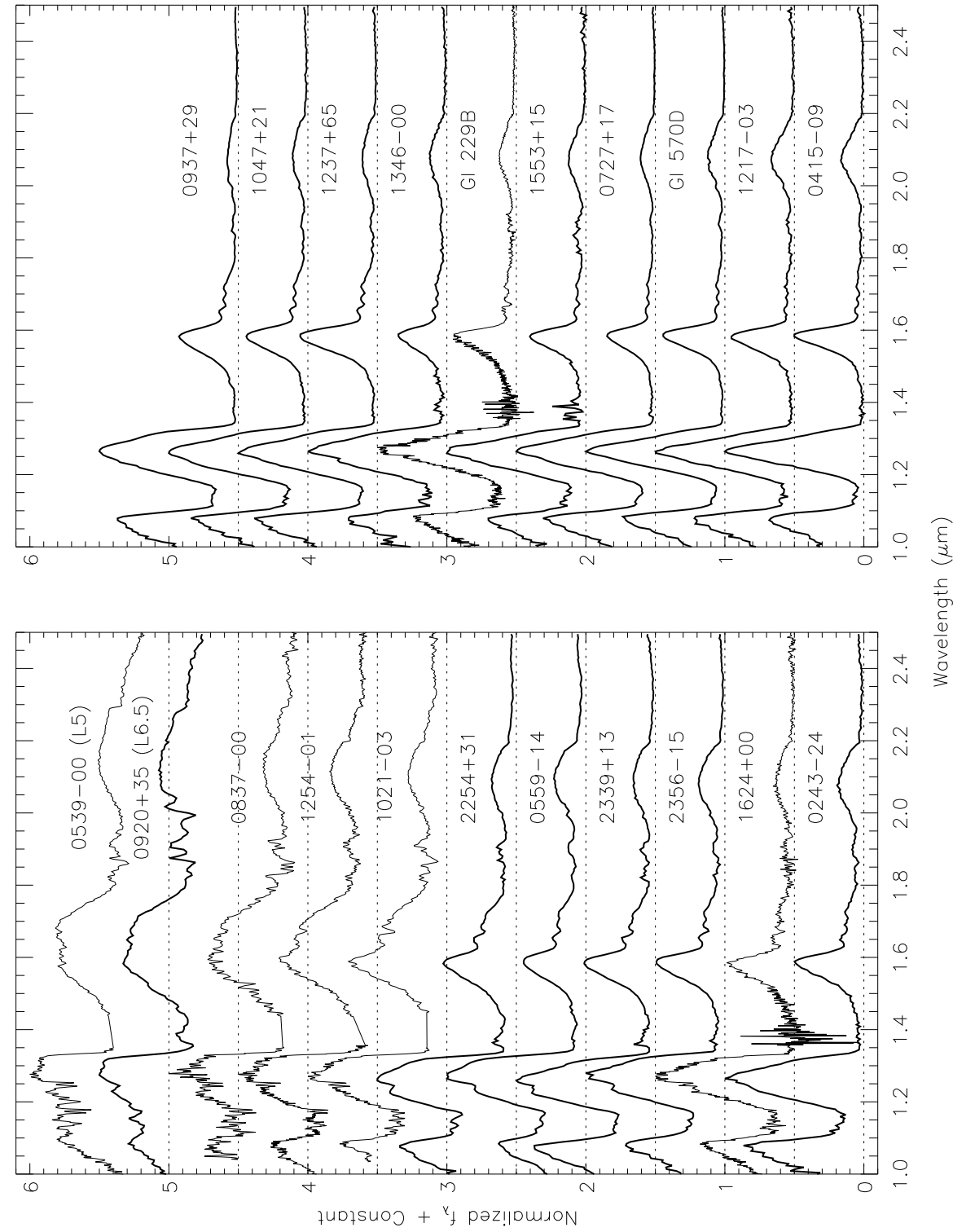

Fig. 4. Near-infrared T dwarf spectra. Data obtained from NIRC observations are shown in thick lines, while (higher resolution) data obtained from the literature 14, 41, 24, are shown as thinner lines. Spectra are normalized at their J-band peaks and offset by a constant. Order is determined visually by comparison of the depths of the $1.15 \mu m \mathrm{H}_{2} \mathrm{O} / \mathrm{CH}_{4}$ and $1.6 \mu m \mathrm{CH}_{4}$ features. 
Adam J. Burgasser, et al.
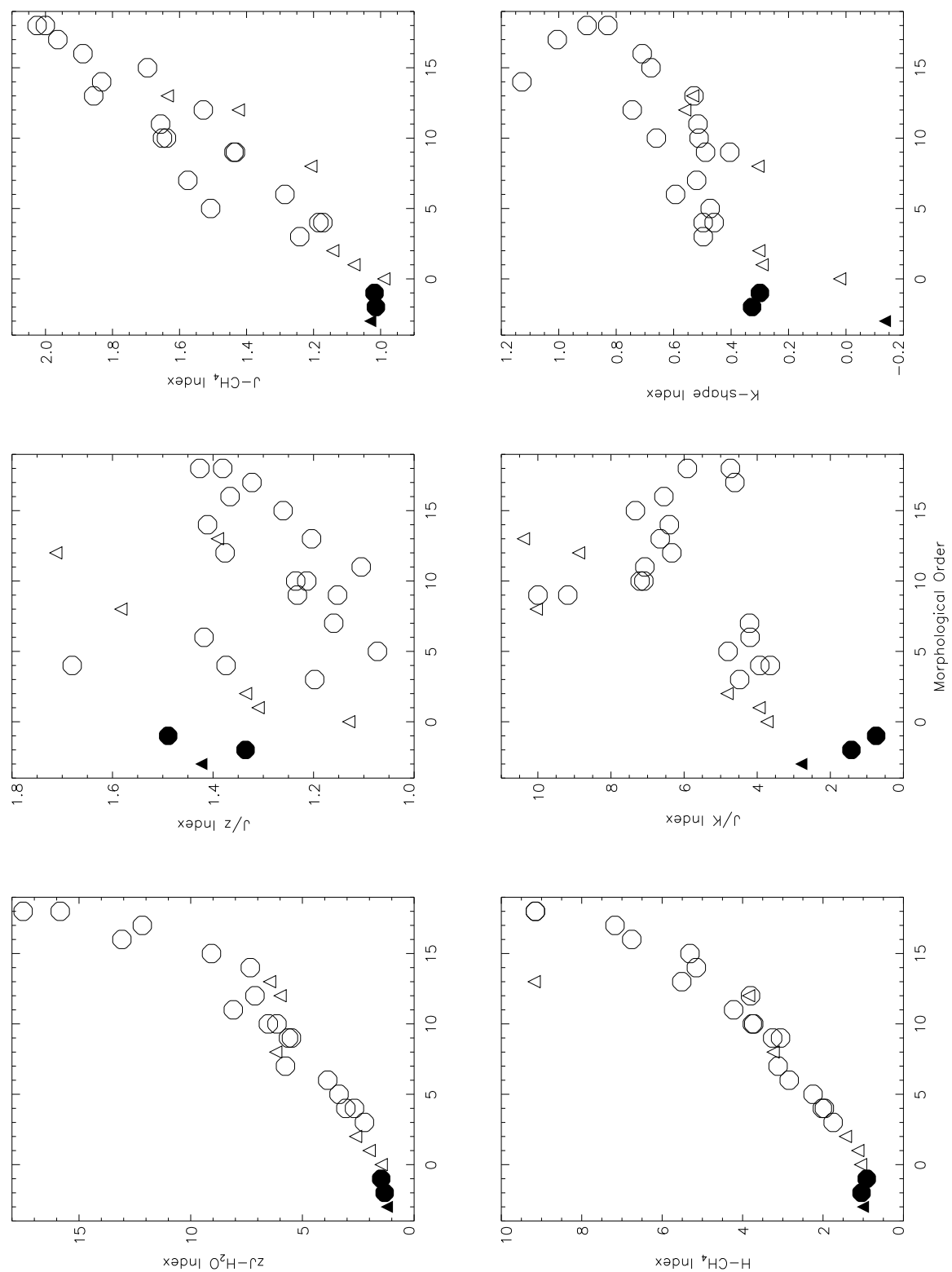

Fig. 5. Near-infrared spectral indices for L (filled symbols) and T dwarfs (open symbols). Measurements of NIRC data are shown as circles, while data obtained from the literature [14, 41 24] are plotted as triangles. T dwarfs are ordered as shown in Fig. 4. Indices are described in Table 2. 

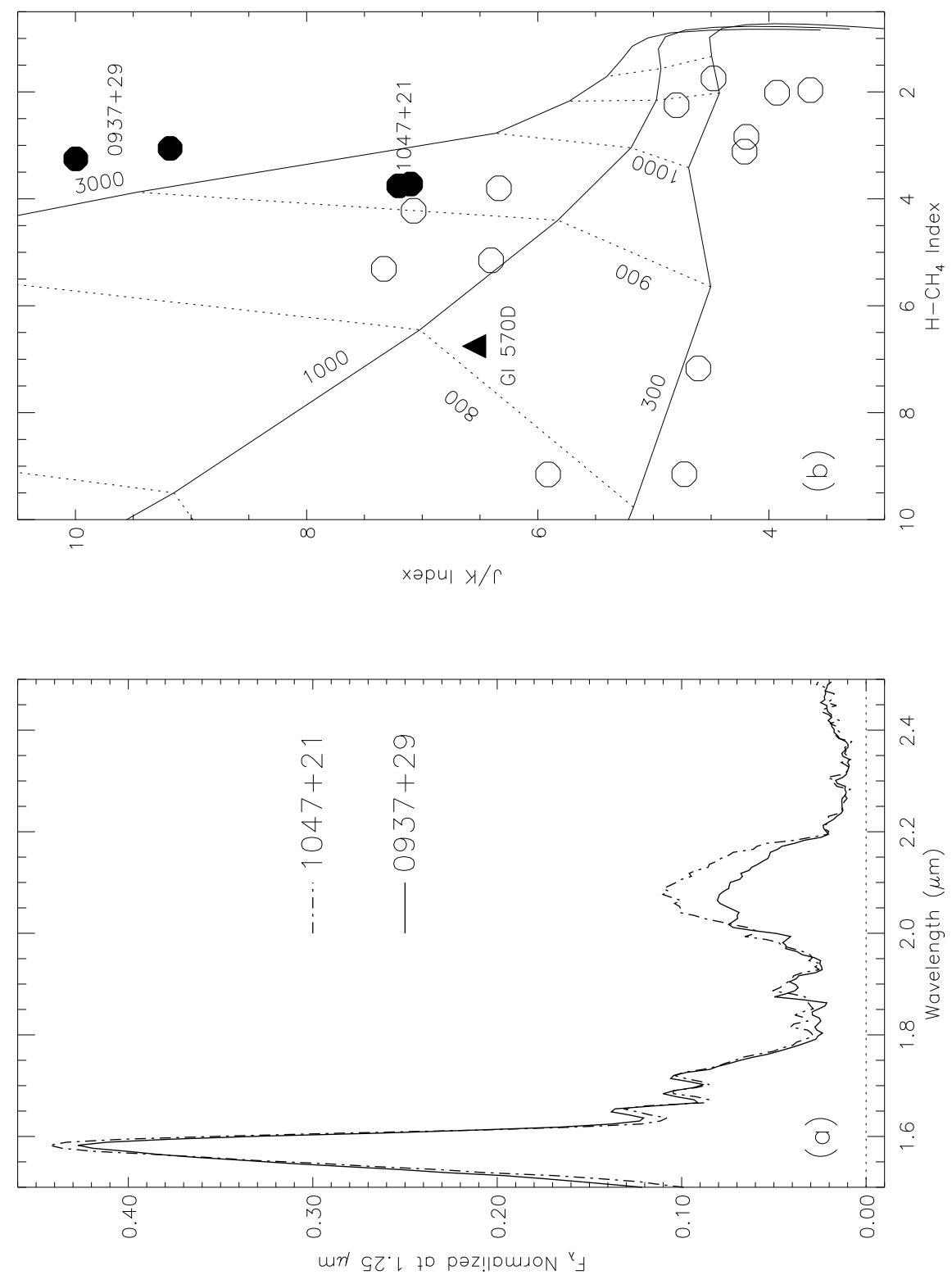

Fig. 6. Spectral estimation of $\mathrm{T}$ dwarf gravities. (a) Spectral comparison between 2MASS $0937+29$ (solid line) and 2MASS 1047+21 (dashed line); both spectra are normalized at their J-band peaks. (b) $\mathrm{H}_{-} \mathrm{CH}_{4}$ index versus $\mathrm{J} / \mathrm{K}$ peak ratio for $2 \mathrm{MASS}$ $\mathrm{T}$ dwarfs and $\mathrm{T}$ dwarf models provided by D. Saumon (priv. comm.) Lines trace out model values for gravities of 300,1000 , and $3000 \mathrm{~m} \mathrm{~s}^{-2}$. 